\title{
A New Invisible Cloak with Broad Stealth Angle Based on Isotropic Material
}

\author{
Zhao Jingbo* \\ Science College \\ Air Force Engineering University \\ Xi'an, China \\ e-mail: chjzjb@163.com \\ * Corresponding Author
}

Du Jun

Institute of Aeronautics and Astronautics Air Force Engineering University

Xi'an, China

Jiang Jiulong

Institute of Aeronautics and Astronautics Air Force Engineering University

Xi'an, China

\begin{abstract}
Study on invisible cloak has attracted considerable attention from all over the world thanks to its potential application value. However, it is still difficult to achieve fully movable invisible cloak due to material and other constrains currently. Here, the design and experimental demonstration of a new invisible cloak for visible light is reported, which is composed of ordinary homogeneous and isotropic glass material, and the ingenious structural design creates an illusion by guiding the light around the hidden object and making it appear on the other side without any deflection. Moreover, the cloak possesses broad oblique viewing angle with perfect stealth effects. Experiments on the designed invisible cloak validate the effectiveness of the proposed cloak structure for visible light.
\end{abstract}

Keywords- Invisible cloak; Structure design; Deflection; Isotropic; Stealth angle

\section{INTRODUCTION}

Research on invisible cloak has made progress in transformation optics[1] and conformal mapping theory[2]. The cloaks include three types, cylindrical3-5, spherical1 and carpet cloak[6-19]. Since the first experimental demonstration of the cylindrical cloak on 2006[3], it is difficult to achieve an isolated invisibility cloak because of the extreme values of the materials and the spatially dependent anisotropic parameters. Linear homogeneous coordinate transformation is used to achieve movable invisible cloak for visible light. The idea on homogeneous

\author{
Yao Hong \\ Science College \\ Air Force Engineering University \\ Xian, China \\ Liu Yijing \\ Science college \\ Air Force Engineering University \\ Xi'an, China
}

Dong Yake

Institute of Aeronautics and Astronautics

Air Force Engineering University

Xi'an, China 


\section{DESIGN OF BASIC INVISIBLE UNIT}

Figure1(a) shows the structure of the unit processed with ordinal glass, which is homogeneous and isotropic organic glass (light transmission rate of $92 \%$, refractive index of 1.49). The The device has two glass flanks included angle 60 degrees. Fig. 1(b) shows stealth effect of the structure. An ordinary pen is placed stealth region (near outside tip) and the background pattern is the letters and logo of Air Force Engineering University. It can be seen from the figure, although stealth object is invisible, the letter 'eer' and part logo of the background pattern has also been hidden at the same time. Thus it can not be called true stealth, but can be applied in some special situation, for example, the shape and color of the background are exactly the same. Fig.1 (c) shows the mechanism analysis of basic invisible unit. The red circle means stealth object. The orange lines with arrowheads mean incident rays. The main reason of the object can be hidden lies to total reflection. As it is known, when light enters from a denser medium into the optically thinner medium, if the incident angle is large to a certain extent, so that when the refraction angle will reach $60^{\circ}$. With increase of the incidence angle, the light is not refracted, and when the light will be reflected, without refraction, this phenomenon is called "total reflection". The total reflection angle of common glass is $38^{\circ}$ for this kind of glass. In the structure of the invisible unit with 60 degree included angle, rays emitted from object are all reflected in inner surface of glass in the range of observation, and do not enter the inside glass, so the object can be invisible. While the same reason that the background pattern is hidden off a block. According to the principle of reversibility of light, after the rays emitted from the inside of the unit pass through the invisible unit, the stealth region formed near the unit tip outside because of refraction, that is to say, the rays emitted from stealth region object can not enter the interior side of unit. But it must be pointed out that, with increasing of the distance between the tip and the object, the incident angle of the rays from object at the inner surface of the glass becomes smaller. When the incident angle is less than total reflection angle, the rays will enter the inside glass, thus observers can see the object or background pattern. The specific mechanism of ray trace can be seen in Fig 1 (d).

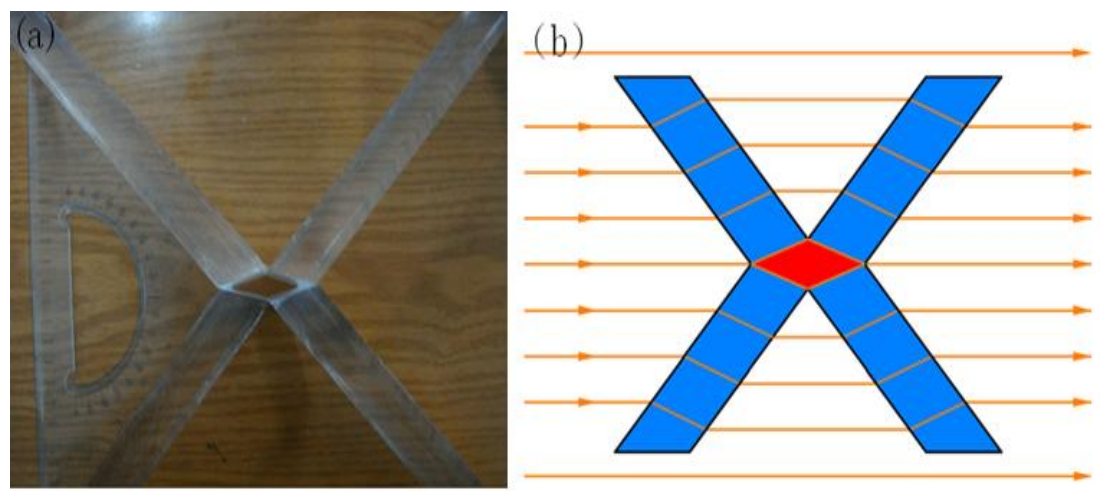

Figure 1. Design and stealth mechanism of the basic invisible unit

In order to solve the narrow stealth angle, a new invisible cloak is designed. Fig. 2 shows the structure and mechanism of the second invisible cloak. Two glass flanks included angle 60 degrees and the hole located in cloak center is stealth region in Fig. 2(a). Fig. 2(b) shows the stealth mechanism. Artful structure design makes the light path round stealth objects, to the other side, trajectory without any offset, realizing the object stealth.

Fig. 3(a) shows the map of the exhibition of experimental setup. A column is put inside the cloak as a hidden object, and the upper part of the column is unwrapped by the cloak for comparison. The cloak with stealth object is placed in air. The letters and the logo of Air Force Engineering University, printed on a paper placed behind the cloak in the $\mathrm{xz}$ plane. To facilitate comparison, a camera is placed in front of the cloak, rotate the cloaks to change viewing angles from $0^{\circ}$ to $30^{\circ}$, then from $0^{\circ}$ to $-30^{\circ}$. The stealth effects for the cloak are shown in Fig. 4. One can see that the background can be seen completely at the viewing angle $0^{\circ}$ (Fig. 3(b)), $30^{\circ}$ (Fig. 3(c)), $+30^{\circ}$ (Fig. 3(d)) respectively. The column object is invisible and the background is visible in viewing angle variation of $\sim 60^{\circ}$ (from $-30^{\circ}$ to $+30^{\circ}$ ). It should be pointed that in Fig. 4(c) and Fig. 4(d), the line is slight thick because of the mismachining tolerance for the cloak. The results indicate that the cloaking performance is valid when viewed at the broad oblique angle range $\sim 60^{\circ}$. 

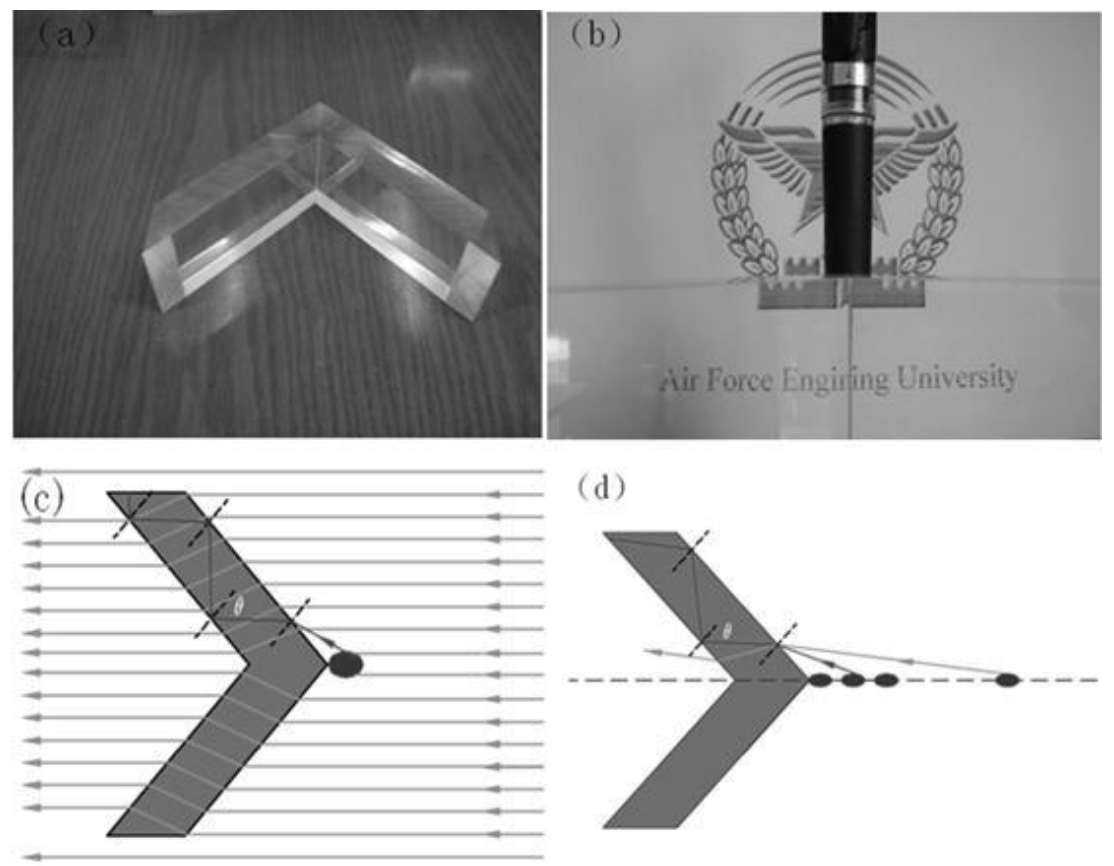

Figure 2. The second invisible cloak (a) The structure of the second invisible cloak (b) The invisible mechanism of the second invisible cloak
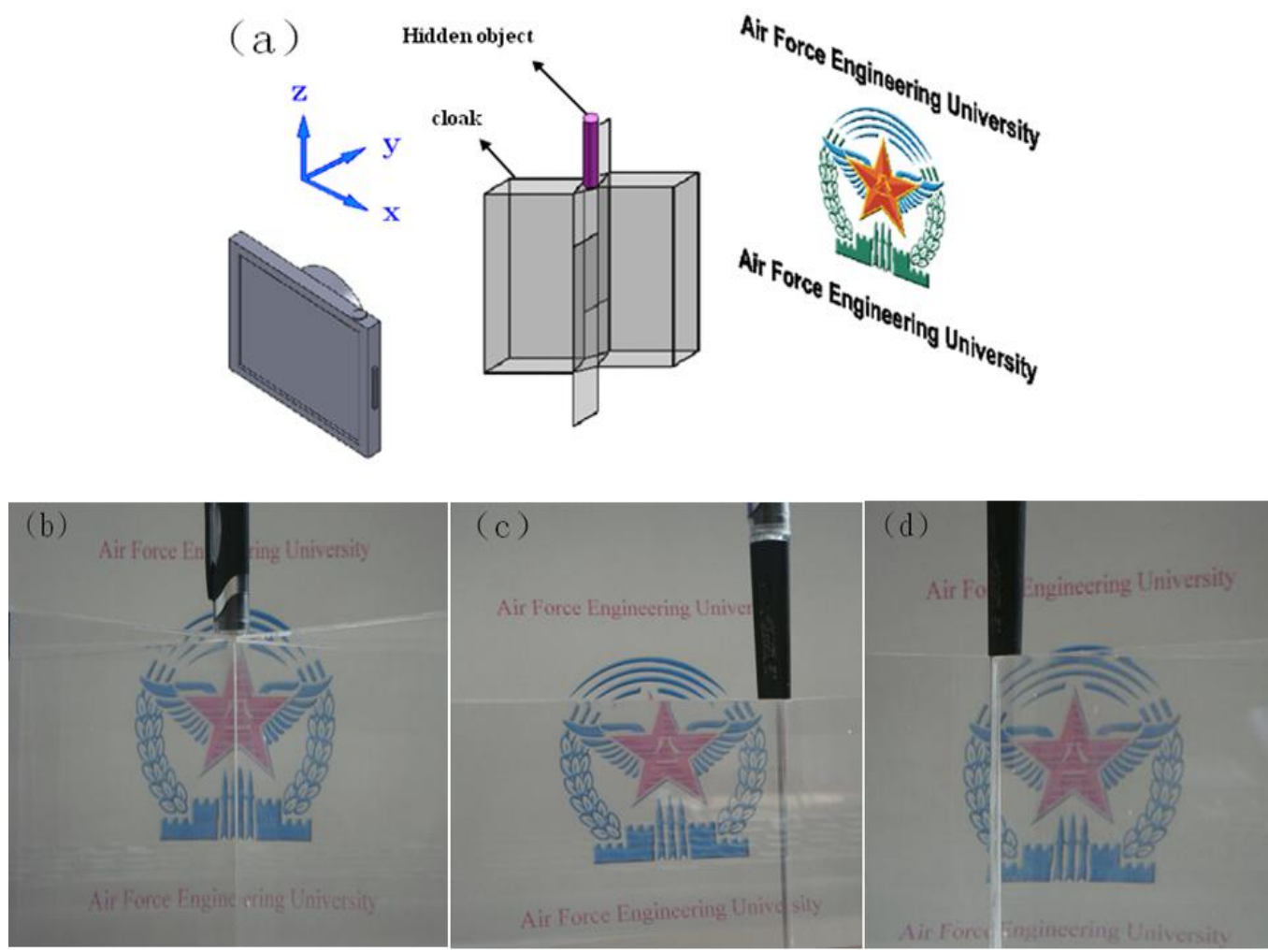

Figure 3. The experiment effect of the second invisible cloak (a) schematic of experiment setup (b) The invisible effect at the viewing angle $0^{\circ}$ (c) The invisible effect at the viewing angle $+30^{\circ}(\mathrm{d})$ The invisible effect at the viewing angle $-30^{\circ}$ 


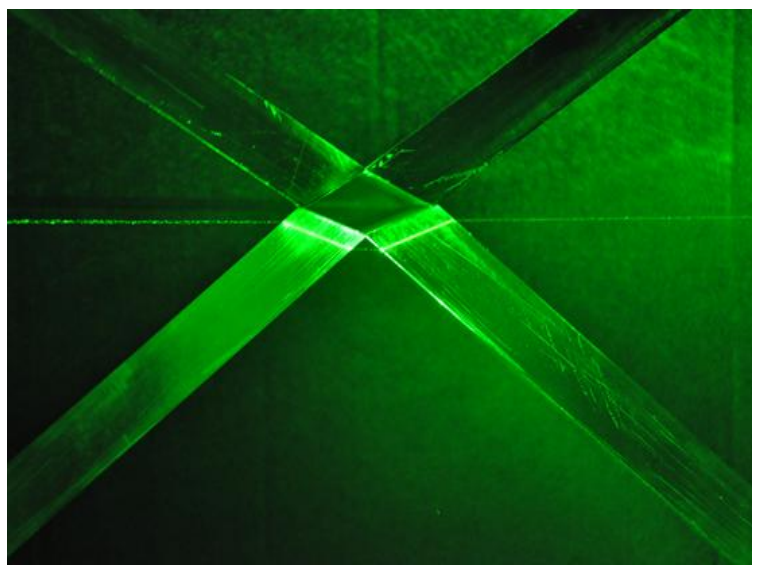

Figure 4. The propagation of the laser beam through the cloak

At the wavelength of $=532 \mathrm{~nm}$ laser irradiation, the light propagation path of the second invisible cloak can be clearly seen, as shown in Figure 3. As it can be seen from the picture, the ray paths are bypassing the stealth region, and are still in the same straight line. The refraction characteristics of laser path further validate the stealth principle and feasibility of the designed invisible cloaks.

The second invisible cloak has three advantages: first, the invisible cloak has broad stealth angle range $\sim 60^{\circ}$. At the angle range, the invisible cloak has perfect stealth effect. Secondly, the invisible cloak can be fabricated using common homogeneous and isotropic glass materials, without using the anisotropic materials and sophisticated processing technology. Thirdly, the invisible cloak can realize the full range of perfect optical camouflage in air environment.

\section{CONCLUSIONS}

In the paper, a new invisible cloak is designed. The stealth mechanism analysis and the experiment have proved the effects of the invisible cloak. The second invisible cloak with broad oblique viewing angle is made of ordinary isotropic glass material, without using sophisticated materials. Therefore, the invisible cloak possesses important value in large-scale engineering application. Meantime, it also has the important enlightenment significance to the non-visible light wave electromagnetic wave stealth.

\section{ACKNOWLEDGMENT}

This work was supported by the National Natural Science Foundation of China (Grant No.11447147,11504429), the National Science Foundation of Shaanxi Province, China(Grant No.2015JQ5155)

\section{REFERENCES}

[1] Pendry, J. B., Schurig,D.\&Smith,D. R.Controlling electromagnetic fields. Science 312, 1780-1782 (2006).

[2] Leonhardt, U. Optical conformal mapping. Science 312 , 1777-1780 (2006).

[3] Schurig, D. et al. Metamaterial electromagnetic cloak at microwave frequencies. Science 314, 977-980 (2006).

[4] Cummer, S. A., Popa, B.-I., Schurig, D., Smith, D. R. \& Pendry, J. Full-wave simulations of electromagnetic cloaking structures. Phys. Rev. E 74, 036621(2006).

[5] Cai, W., Chettiar, U. K., Kildishev, A. V. \& Shalaev, V. M. Optical cloaking with metamaterials. Nat. Photon. 1, 224-227 (2007).

[6] Li, J. \& Pendry, J. B. Hiding under the carpet: a new strategy for cloaking. Phys. Rev. Lett. 101, 203601 (2008).

[7] Liu, R. et al. Broadband ground-plane cloak. Science 323, 366-369 (2009).

[8] Valentine, J., Li, J., Zentgraf, T., Bartal, G. \& Zhang, X. An optical cloak made of dielectrics. Nat. Mater. 8, 568-571 (2009).

[9] Gabrielli, L. H., Cardenas, J., Poitras, C. B. \& Lipson, M. Silicon nanostructure cloak operating at optical frequencies. Nat. Photon. 3, 461-463 (2009).

[10] Ma, H. F. \& Cui, T. J. Three-dimensional broadband ground-plane cloak made of metamaterials. Nat. Commun. 1, 21 (2010).

[11] Ergin, T., Stenger, N., Brenner, P., Pendry, J. B. \&Wegener,M. Three dimensional invisibility cloak at optical wavelengths. Science 328 , 337-339 (2010).

[12] Zhang, B., Luo, Y., Liu, X. \& Barbastathis, G. Macroscopic invisibility cloak for visible light. Phys. Rev. Lett. 106, 033601 (2011).

[13] Chen, X., Luo, Y., Zhang, J., Jiang, K., Pendry, J. \& Zhang, S. Macroscopic invisibility cloaking of visible light. Nat. Commun. 2, 176 (2011).

[14] Ergin, T., Fischer, J. \&Wegener,M. Optical phase cloaking of 700 $\mathrm{nm}$ light waves in the far field by a three-dimensional carpet cloak. Phys. Rev. Lett. 107, 173601(2011).

[15] Lee, J. H. et al. Direct visualization of optical frequency invisibility cloak based on silicon nanorod array. Opt. Express 17, 12922-12928 (2009).

[16] Gharghi,M. et al. A carpet cloak for visible light. Nano Lett. 11, 2825-2828 (2011)

[17] Tamma, V. A., Blair, J., Summers, C. J. \& Park, W. Dispersion characteristics of silicon nanorod based carpet cloaks. Opt. Express 18, 25, 25746-25756 (2010).

[18] Zhang, J., Liu, L., Luo, Y., Zhang, S. \& Mortensen, N. A. Homogeneous optical cloak constructed with uniform layered structures. Opt. Express 19, 9, 8625-8631(2011).

[19] Xu, X. et al. Broad band invisibility cloak made of normal dielectric multilayer. Appl. Phys. Lett. 99, 154104 (2011).

[20] Xi, S., Chen, H.,Wu, B.-I. \& Kong, J. A. One directional perfect cloak created with homogeneous material. IEEE Microw. Wirel. Compon. Lett. 19, 131-133 (2009)

[21] Chen, H. S., Zheng, B. Broadband polygonal invisibility cloak for visible light. Sci. Rep. 2, 255(2012).

[22] Chen, H. S., Zheng B., Shen L., Wang H. P., Zhang X.M., Nikolay I., Zheludev and Zhang B.L. Ray-optics cloaking devices for large objects in incoherent natural light. Nat. Commun. 4:2652(2013) 